\title{
Computerised cognitive behaviour therapy (cCBT) as treatment for depression in primary care (REEACT trial): large scale pragmatic randomised controlled trial
}

The original trial registration for this Research paper (BMJ 2015;351:h5627, doi:10.1136/bmj.h5627), the REEACT trial (http://www.controlled-trials.com/ISRCTN91947481), included depression as a primary outcome, measured in two ways: "Depression severity and symptomatology as measured by a validated self-report measure (the Patient Health Questionnaire [PHQ-9]) and the International Classification of Diseases (ICD-10) depression score at four months." The original protocol defined the primary outcome measure as "depression severity and symptomatology as measured by the PHQ-9 and a self-report computer-administered diagnostic interview (CIS-R) at four months." Before the start of the trial, the primary outcome measure was modified to consist of only the PHQ-9 at four months. We did not collect ICD diagnosed depression using a standardised interview schedule. The public document detailing all revisions can be accessed in the full report at http: //www.journalslibrary.nihr.ac.uk/hta/volume-19/issue-101\# chapters/2. One of our outcomes was a measure of quality adjusted life years, which is published in the full economic evaluation at http://www.journalslibrary.nihr.ac.uk/hta/volume19/issue-101\#chapters/4.

Cite this as: BMJ 2016;352:1195

๑ BMJ Publishing Group Ltd 2016 